\title{
Una nueva categoría docente en la universidad española del franquismo: la aparición en escena del profesor agregado
}

\section{A new teaching category at the Spanish University of Franco's regime: the appearance on the scene of the senior lecturer}

Sara GONZÁLEZ GÓMEZ

Universidad de las Islas Baleares

Recibido: Mayo 2014

Evaluado: Septiembre 2014

Aceptado: Septiembre 2014

\section{Resumen}

La Ley sobre estructura de las facultades universitarias y su profesorado aprobada el 17 de julio de 1965 colocó la figura del profesor agregado sobre el escenario universitario. Durante los años sesenta, el proceso de gestación, aprobación y posterior integración de esta nueva figura en la pirámide académica no escapó a la controversia, dando lugar a un fenómeno complejo al que, sin embargo, no se han dedicado estudios exhaustivos que revelen cómo, cuándo, por qué y bajo qué condiciones apareció este docente en la universidad española del franquismo.

El presente trabajo pretende aportar al lector una visión general sobre el proceso de concreción de la figura del agregado, el marco normativo que definitivamente estableció esta nueva categoría docente así como las disposiciones que se encargaron de regular los procesos de concurso-oposición. Asimismo, se analizarán las primeras dotaciones de agregados aprobadas y la distribución de las mismas por universidades, facultades y estudios. Para terminar, se realiza un primer acercamiento a las posibles motivaciones que condujeron a la creación de esta nueva figura docente en una universidad aquejada de graves problemas que esperaban a una solución que venía demorándose sine die.

Palabras clave: universidad española, pirámide docente, profesor agregado, franquismo.

\begin{abstract}
The Law of 17 July 1965 on the structure of university faculties and their teaching staff placed the figure of the university senior lecturer on stage. During the sixties, the gestation process, adoption and subsequent integration of the new figure in the academic pyramid did not escape controversy, leading to a complex phenomenon to which, however, non comprehensive studies have been devoted to reveal: how, when, why and under which conditions this teacher appeared in the Spanish University of Franco's regime. This paper aims to provide an overview of the process of materialization of the senior lecturer, the legislative framework that definitely established this new educational category and the provisions that were in charge of regulating


the processes to get tenure. Also, the first approved aggregate endowments and their distribution among universities, colleges and studies will be discussed. Finally, it was a first approach to the possible motivations that led to the creation of this new educational category in a university afflicted by serious problems waiting for a solution that had been delayed sine die.

Keywords: spanish university, teaching pyramid, senior lecturer, Franco’s regime.

Durante la década de los sesenta, la universidad española oteaba el futuro sin posibilidad de vislumbrar una perspectiva demasiado halagüeña. El progresivo aumento del alumnado, la escasez de espacios y recursos materiales, la falta de personal docente, la reducida dedicación a labores investigadoras o el clima de agitación estudiantil, entre otras muchas cosas, habían conducido a la institución hacia una coyuntura realmente compleja. A todo ello habría que sumar el mantenimiento de un modelo universitario tradicional, de corte napoleónico, marcadamente centralista y que habría demostrado sobradamente su incapacidad para abrir nuevas vías conducentes a la evolución y modernización del sistema.

El escenario era realmente desalentador, pero lo cierto es que no todo fueron sombras en la universidad de la época. Al compás de las reformas económicas que aspiraban a propiciar el proceso de desarrollismo español; junto a la progresiva apertura de fronteras al exterior; con la aparición de una representación social mucho más amplia en los colectivos estudiantiles y, especialmente, por la influencia o las presiones ejercidas por algunos organismos internacionales, el mando ministerial del momento comenzó a adoptar algunas medidas que cambiaron, aunque ya adelantamos que no radicalmente, el panorama universitario español.

Una de esas medidas llegó a mediados de los sesenta con la promulgación de la Ley de 17 de julio de 1965 sobre estructura de las facultades universitarias y su profesorado. Con ella, tras intensos y prolongados años de debate, aparecieron en el escenario universitario dos nuevos protagonistas: el profesor agregado y el extraordinario. Asimismo, irrumpió en escena una nueva forma de organización académica a partir de departamentos, en un intento por comenzar a desplazar el sistema de cátedras, convertidas en auténticos adarves de conocimiento y poder, hacia un sistema de trabajo conjunto entre profesores de diferentes categorías, capaz de generar el espacio idóneo para el cultivo de la ciencia y la difusión de la investigación.

El alcance real de estas medidas, bastante limitado por ejemplo en la articulación real de los departamentos, merece un estudio detallado y pormenorizado que todavía hoy está pendiente de efectuarse. La nueva gradación de la pirámide docente y un novedoso tipo de organización departamental entraron a formar parte de la universidad española de mediados de los sesenta despertando opiniones de todo tipo y cambiando, en cierta medida, una parte sustancial del sistema universitario hasta entonces vigente.

En las últimas décadas se han publicado excelentes trabajos en los que se aborda el estudio de la universidad del franquismo (v. gr. Carreras Ares y Ruiz Carnicer, 1991; Hernández Sandoica, 2002; Claret Miranda, 2006; Hernández Sandoica, Ruiz Carnicer y Baldó Lacomba, 2007; Rodríguez López, 2002; González Gómez, 2013; SEDHE, 1998), de la que conocemos en profundidad algunas cuestiones -modelo educativo, 
marco normativo, procesos de depuración, movilización estudiantil, etc.- pero de la cual, sin duda, seguimos ignorando numerosos aspectos concretos de su vida interna así como los entresijos de su funcionamiento. Una de esas cuestiones que permanece esperando al estudio detallado es la que nos ocupa en este trabajo: la figura de los agregados.

Existen trabajos a través de los cuales se han estudiado los procesos de oposición a cátedras durante la dictadura (Blasco Gil, 2010; Peláez, 2008; Martínez Neira, 2003) que nos aportan una información general de referencia, y se han podido localizar algunas breves alusiones a este tipo de profesores agregados en artículos publicados por Josep Carreras (2003) y Juan Luis Rubio Mayoral (2013). En ambos casos se analiza el contenido legal que se encargó de definir y delimitar las principales categorías docentes universitarias -el acceso, los procedimientos de concurso-oposición o la constitución de tribunales- durante la dictadura. Por su parte, Julio Carabaña (2003) hace referencia a los agregados a través de un documento en el que reflexiona sobre la carrera del profesor universitario.

Pocas más son las referencias localizadas sobre el asunto de los agregados. En consecuencia, entendemos que esta cuestión reviste un especial interés dado que nos permite desvelar un aspecto particular y destacado de la universidad de la época. A través de este trabajo, en primer lugar, se pretende aportar una visión general sobre el proceso de gestación de la figura del agregado, utilizando para ello los debates que generó el asunto en Consejo de Rectores. En segundo lugar, se procederá a concretar y analizar el marco normativo así como las disposiciones que se encargaron de regular los procesos de concurso-oposición. A continuación se analizarán las primeras dotaciones de agregados aprobadas así como su distribución. Finalmente, se realizará un inicial y básico acercamiento a las posibles motivaciones que condujeron a la creación del agregado.

\section{Proceso de gestación de la categoría del agregado}

Las ratios profesor/alumno comenzaron a desbordarse a partir de los años sesenta en la mayor parte de las universidades españolas. El número de universitarios crecía de forma progresiva a principios de la década y la tendencia se aceleraba de forma notable a partir de la medianía de los sesenta. Sin embargo, el ritmo de incremento del profesorado no seguía la misma tendencia, generando un importante desfase numérico entre uno y otro colectivo y recrudeciendo los problemas del sistema universitario.

Todo ello provocó que la reorganización del profesorado se convirtiera en un asunto de trascendental importancia durante la época y en una problemática que debía ser atendida con la mayor celeridad posible. De hecho, todas las universidades dejaron constancia a través de sus respectivos rectorados, en los que se recibían con mucha frecuencia propuestas y reclamaciones efectuadas desde sus juntas de facultad, de los problemas que este asunto estaba generando en el día a día académico.

Una de las primeras medidas adoptadas por el Ministerio para atajar la problemática del desfase de las ratios profesor-alumno consistió en la creación de la categoría docente del agregado, figura intermedia entre el catedrático ordinario y el profesor 
adjunto. A la adopción oficial de la medida en el año 1965 precedió el debate, cargado de cierta controversia, en las diversas sesiones efectuadas regularmente por el Consejo de Rectores. La primera referencia a esta figura la encontramos en la sesión celebrada el 12 de febrero de 1960, en la cual ya se sugería la conveniencia de modificar el artículo 56 de la Ley sobre ordenación de la universidad española de 1943 (LOU) introduciendo la categoría del agregado.

En aquel momento, la distinción entre éstos y los profesores adjuntos se esquematizaba en cuatro fases ${ }^{1}: 1^{\mathrm{a}}$ ) Profesor adjunto, con título de licenciado y cuatro años de permanencia en el cargo; $2^{\mathrm{a}}$ ) Prórroga de cuatro años en el ejercicio del cargo, exigiéndose para la misma el título de doctor; $3^{\mathrm{a}}$ ) Prórroga de cuatro años en el ejercicio del cargo; $4^{\mathrm{a}}$ ) Al terminar el plazo de doce años que comprenden las tres fases anteriores, el profesor adjunto cesaría como tal, pero podría pasar a la categoría de agregado, mediante determinadas condiciones y con carácter permanente. El profesor agregado adquiriría la condición de funcionario con todas las ventajas inherentes a dicha categoría.

A partir de este planteamiento preliminar se inició un proceso de delimitación de una nueva figura que todavía tardaría un lustro en incorporarse definitivamente a la universidad. Inicialmente, algunos rectores se preguntaron por las funciones reales que vendría a ostentar dicho docente; tal es el caso del rector de la Universidad de Madrid, Segismundo Royo-Villanova, que aprovechaba una de esas sesiones para interrogar al director general de enseñanza universitaria sobre la verdadera entidad de ese profesor, recibiendo la siguiente contestación:

«Podría considerarse como el Vicecatedrático, al que en todo caso correspondería encargarse del desdoblamiento de la Cátedra. Será el profesor cualificado que, bajo la dirección del Catedrático, regentará las Cátedras desdobladas; sustituirá al Catedrático en sus ausencias, enfermedades, etc. y será encargado de las Cátedras vacantes»².

Del estudio del debate propiciado en la sesión del Consejo de Rectores citada se deduce un evidente establecimiento de dos bandos contrarios. Por un lado, la mayor parte de los rectores que consideraban acertada la propuesta de este nuevo tipo de docente, aunque insistían en la importancia de hacer constar en la normativa, de forma muy clara, la dependencia de éste de la dirección del catedrático; por otro lado, una parte minoritaria que entendía que lo realmente necesario era mejorar la inestable situación del profesor adjunto -por su temporalidad y falta de permanencia- y proceder a una remuneración acorde al trabajo que desempeñaba. Así lo defendían José Beltrán de Heredia, rector de la Universidad de Salamanca (1956/60), y Valentín Silva Melero, rector de la Universidad de Oviedo (1954/60), expresando que era preciso distinguir entre el interés de la universidad y el de los actuales profesores adjuntos, entendiendo

\footnotetext{
${ }^{1}$ Cfr., ArChivo HistóRICo de la Universidad de SALAmANCA -en adelante AUSA-, Consejo de Rectores, sesión de 12 de febrero de 1960 (turno de mañana), AC 3275/8.

${ }^{2}$ Cfr., AUSA, Consejo de Rectores, sesión de 12 de febrero de 1960 (turno de mañana), AC 3275/8.
} 
que la situación de estos docentes no se resolvería con la creación de la figura del agregado sino otorgando un carácter permanente a los propios adjuntos.

Surgía aquí un complicado asunto universitario que venía reclamando una solución desde tiempo atrás: la mejora de las condiciones del profesorado adjunto. Debemos recordar que dentro de las facultades universitarias, el catedrático constituía el único personal docente con nombramiento de carácter vitalicio. A su vez, las cátedras se transformaban en grandes adarves o trincheras personales en las que se realizaba un trabajo, en la mayor parte de los casos, de carácter individual. El resto del profesorado (adjuntos, ayudantes, lectores, etc.) asumía una responsabilidad docente muy amplia, ocupándose de numerosas labores docentes y de carácter investigador, pero bajo unas condiciones salariales y de reconocimiento social verdaderamente limitadas si se comparan con las de los catedráticos.

Sin embargo, la postura de Torcuato Fernández-Miranda, director general de enseñanza universitaria, difería de la del rector de la universidad salmantina. Para aquél, el proyecto de creación de la figura del agregado obedecía a la conveniencia de no otorgar un carácter permanente a los adjuntos; por ello surgían aquéllos, con la ventaja de estimular la labor del adjunto, motivándole a la consecución de una plaza de este tipo para adquirir esa estabilidad profesional. A su juicio, esta era la única solución para el actual profesorado.

Por su parte, Segismundo Royo-Villanova, rector de la Universidad de Madrid (1956/64), entendía que el profesor adjunto no debía tener una categoría inferior al agregado, pues la propia LOU de 1943 encargaba automáticamente a dicho profesorado las cátedras vacantes.

Lo cierto es que, inicialmente, la idea del profesor agregado surgía casi exclusivamente como una consecuencia de la necesidad de contar con docentes que se encargaran del desdoblamiento de cátedras al que comenzaba a obligar en muchos casos el aumento del alumnado universitario y que ya no podían cubrir esos adjuntos. Pero el asunto, inmediatamente, pasó a un segundo plano en el que se orientó el sentido de su creación hacia un sistema para la selección del profesorado adjunto, pues no habría plazas de agregados para todos, que serviría para motivarles y ofrecerles una amplia perspectiva de consolidación.

El proyecto de Ley de reorganización del profesorado universitario continuó gestándose en años sucesivos. En sesión del Consejo de Rectores de 28 de febrero de $1963^{3}$, el debate se centró en las posibles exigencias que deberían cumplir los aspirantes. Manuel Batlle Vázquez, rector de la Universidad de Murcia (1944/75), propuso que para concurrir a la oposición debería acreditarse la prestación de servicios docentes en la universidad durante un periodo mínimo de dos años. Alfonso Balcells Gorina, a la sazón rector de la Universidad de Salamanca (1960/68), mantenía la misma postura alegando que, de este modo, se evitarían improvisaciones en esta

\footnotetext{
${ }^{3}$ Cfr., AUSA, Consejo de Rectores, sesión de 28 de febrero de 1963, AC 3275/10.
} 
materia. La experiencia docente sería años más tarde uno de los aspectos que se tendría en cuenta en la selección.

Otro de los asuntos abordados fue la posible denominación de este nuevo profesorado, sugiriéndose los nombres de profesor extraordinario, numerario o agregado numerario, designaciones que finalmente serían desestimadas.

Asimismo, se debatió sobre la conveniencia de la obligatoria dedicación exclusiva que se proponía exigir a éstos, cuestión que el director general de enseñanza universitaria consideraba imprescindible. En este punto, Balcells llamó la atención de los rectores proponiéndoles la introducción de diferencias entre las facultades de Medicina y el resto. Estamos aquí ante una posición que se repitió en numerosos casos con rectores de la rama médica, no sólo en este asunto sino también en otras cuestiones universitarias -por ejemplo, se observa la misma posición en cuanto a la regulación de la dedicación exclusiva del catedrático a la universidad-. De hecho, en diversas ocasiones, las propuestas de reforma presentadas durante este periodo tenderían a realizar distinciones entre los estudios médicos y los desarrollados en el resto de facultades universitarias.

En este caso, el director general no se mostró de acuerdo con la idea del rector de Salamanca, mencionando que, si no se llegaba al principio radical de la dedicación exclusiva a la universidad por parte de todos los profesores numerarios, se perpetuaría la situación irregular que se venía produciendo desde hacía muchos años -el grave problema del absentismo docente, las cátedras de las que se ocupaban adjuntos por estar los catedráticos encargados de ellas ocupados en otras labores y cargos, etc.-.

El resto de rectores parecían también partidarios de no introducir esta diferenciación; de hecho, José Corts, rector de la Universidad de Valencia (1952/67), expresaba en su exhortación que el ejercicio profesional, indudablemente, producía el abandono de la función docente.

Como vemos, la polémica estaba servida. En septiembre de 1963 ya se habían redactado cuatro proyectos referentes a la cuestión del profesorado y, aquel mismo año, llegó a todas las universidades españolas una copia del proyecto final de líneas fundamentales de reestructuración del profesorado. En este documento se señalaba el procedimiento de entrada del agregado, mediante oposición nacional, su carácter permanente en el cargo y la exigencia de dedicación exclusiva a la universidad. Asimismo, se contemplaban en sus derechos obvencionales y en sus haberes la percepción de una cantidad que no sería inferior al $75 / 80 \%$ de lo que en esos momentos percibían los catedráticos. Tras un cierto periodo de servicio ininterrumpido (de cinco a ocho años) sería elegible, por concurso nacional, para cubrir las vacantes que se fueran produciendo entre catedráticos numerarios ${ }^{4}$.

El asunto del agregado no volvió a salir a colación en Consejo de Rectores durante los dos años anteriores a su aprobación oficial, centrándose ahora el debate en la nueva

\footnotetext{
${ }^{4}$ AUSA, Copia del proyecto de líneas fundamentales de reestructuración del profesorado universitario, 1963, 10204/11.
} 
organización de tipo departamental que, por su parte, despertó minoritarias adhesiones y, sobre todo, grandes reticencias entre los catedráticos españoles.

Definitivamente, la figura del profesor agregado resultó aprobada por Ley de 17 de julio de 1965. Veremos a continuación, a partir del análisis de dicha legislación y de las disposiciones posteriores que se encargaron de su concreción, a qué condiciones quedaron sujetas finalmente estas plazas.

\section{Ley de 17 de julio de 1965 sobre estructura de las facultades universitarias y su profesorado y disposiciones posteriores}

El nuevo marco normativo aprobado en 1965 introdujo una serie de medidas encaminadas a generar ciertas modificaciones fundamentales en el ámbito universitario a partir, básicamente, de la creación de tres nuevas figuras académicas: el profesor agregado, el profesor extraordinario y el departamento. Tal y como se recoge en el prólogo al articulado, el crecimiento del alumnado exigía no sólo el adecuado acondicionamiento de espacios y el oportuno incremento de los medios didácticos sino también, y fundamentalmente, la promoción de un profesorado en número suficiente para que la relación docente-discente se mantuviera así en unos términos adecuados para asegurar la eficacia del sistema de enseñanza-aprendizaje.

Una aspiración con tintes utópicos si tenemos en cuenta la gran cantidad de problemas que aquejaban a la universidad española en esos momentos y que no podían atajarse con una única medida que no actuaba en la raíz de la mayor parte de ellos. Sin embargo, tal y como se recogía en aquel prolegómeno, el problema planteado en la universidad no residía únicamente en la adopción de medidas de carácter cuantitativo, sino que sobrevenía la necesidad de reestructurar la estratificación del cuerpo docente:

«La estructura de la Cátedra, como la diferenciación del Profesorado, resultan hoy excesivamente limitadas. La realidad actual, y sobre todo, en relación con ella, el deber de proporcionar a nuestros estudiantes un clima más universal en su formación, obligan a una reconsideración de la situación presente en este aspecto fundamental de la vida universitaria» ${ }^{5}$.

En teoría, atendiendo a estas razones, el régimen optó por crear la figura del agregado, un profesor universitario de rango superior, con dedicación plena a la universidad y sometido a la disciplina del catedrático-jefe del departamento al que quedara adscrito. Su puesto vino a considerarse como el paso previo para la consecución de una cátedra, concretándose en la Ley que el paso ulterior a catedrático se efectuaría entre agregados que cumplieran una serie de características que se fijaron también en la normativa (haber cumplido como mínimo cinco años de servicio activo como agregados ${ }^{6}$ y presentar un currículum vitae con la relación de sus trabajos y

\footnotetext{
${ }^{5}$ Ley 83/1965, de 17 de julio, sobre estructura de las Facultades Universitarias y su Profesorado, BOE ${ }^{\circ}$ 173 de 21 de julio de 1965.

${ }^{6}$ En las disposiciones transitorias, sin embargo, se contempló la reducción de ese periodo a sólo dos años para aquéllos que a la promulgación de la Ley hubieran sido profesores adjuntos por oposición,
} 
publicaciones así como una memoria comprensiva de sus directrices de investigación y del programa, método y fuentes para la enseñanza de la disciplina objeto del concurso).

La aparición del concepto organizativo del departamento junto a la del profesor agregado hizo pensar entonces que el mayor volumen de trabajo que asumiría el primero facilitaría la superación del agregado en su marcha ascendente hacia la cátedra y crearía en él un hábito de entrega a la vida universitaria, objetivo esencial de toda renovación.

El acceso a este nuevo cuerpo se realizaría mediante concurso-oposición de ámbito nacional. Los candidatos deberían reunir unos requisitos mínimos: poseer el título de doctor; acreditar una experiencia docente o investigadora de al menos tres cursos completos o bien ser catedráticos de centros docentes de grado medio y haber completado esos tres cursos; y ser presentados mediante un escrito, firmado por un catedrático de universidad o escuela técnica superior, e informado por la Junta de Facultad o de Profesores respectivamente, o por el CSIC en caso de investigadores del mismo o pertenecientes a centros de investigación nacionales o extranjeros, en el que se explicaran sus cualidades y la labor realizada.

El reglamento de los concursos-oposición para la provisión de plazas de profesores agregados fue aprobado un año después por Orden de 25 de junio de 1966. Mediante esta nueva disposición se fijó en Madrid la verificación de estos procedimientos y se adjudicaron como condiciones necesarias para concurrir a ellos las siguientes: a) Ser español; b) No hallarse el aspirante incapacitado para ejercer cargos públicos; c) Adhesión a los principios fundamentales del Movimiento Nacional y demás Leyes fundamentales del Reino; d) Poseer el título de doctor en facultad o escuela técnica de grado superior ${ }^{7}$.

El plazo para la presentación de solicitudes sería de treinta días hábiles contados a partir de la publicación de la convocatoria en el BOE. Una vez publicadas las listas de admitidos y excluidos en cada uno de los concursos, el Ministerio sería el encargado de designar el tribunal responsable de juzgarlos. Dicho procedimiento de designación se había regulado previamente, mediante Orden de 30 de mayo de 1966, en la cual se estableció lo siguiente:

a) El presidente del tribunal sería designado por el Ministerio de Educación Nacional entre aquellos que ostentaran la condición de miembros del Consejo Nacional de Educación (CNE), de las Reales Academias, del CSIC o bien la de ser o haber sido rector de universidad.

b) Dos vocales catedráticos ordinarios de universidad de la misma asignatura convocada a concurso-oposición que serían designados automáticamente en turno de rotación en el orden de antigüedad en la relación de catedráticos, la que se

colaboradores o investigadores del CSIC por oposición o concurso, o catedráticos de enseñanza de grado medio, todos ellos con tres años de ejercicio continuado.

${ }^{7}$ Orden de 25 de junio de 1966 por la que se aprueba el reglamento para los concursos-oposiciones para la provisión de plazas de profesores de universidad, $B O E \mathrm{n}^{\circ} 157$ de 2 de julio de 1966. 
consideraría dividida en dos partes iguales a estos efectos y para designar un vocal de cada una de ellas.

c) Un vocal catedrático ordinario de universidad de la misma asignatura convocada a concurso-oposición que sería designado por el ministro a propuesta del rector de la universidad a que correspondiera la vacante y que debería formularse una vez oída la junta de facultad.

d) Un vocal, catedrático o no, especializado en la disciplina o en materias similares que sería designado por el ministro a propuesta en terna del CNE.

El examen de oposición constaría de 6 ejercicios eliminatorios: 1) Defensa del currículum; 2) Defensa del concepto, método y programa de la asignatura; 3) Lección magistral con desarrollo de un tema durante una hora; 4) Preparación (4 horas) y defensa de un tema elegido por el tribunal de entre los 10 escogidos por el candidato mediante procedimiento de «insaculación»; 5) Ejercicio práctico; 6) Ejercicio escrito y oral de defensa de un tema a elección del tribunal.

Una vez superado este periplo de pruebas, el candidato vencedor pasaría a asumir labores docentes, investigadoras y examinadoras, de acuerdo con las exigencias de la facultad, el departamento y la cátedra. Se ocuparía, al menos, de un curso o grupo desdoblado de la asignatura a la que quedara sujeto.

Asimismo, estaría obligado al deber de residencia y a la dedicación exclusiva, haciendo referencia en este punto a los modelos seguidos por universidades extranjeras. De hecho, esta alusión al exterior en cuanto a dedicación plena y obligada a la universidad, que por primera vez se exigía directamente a todo aquel que ocupara este tipo de plazas y no como algo voluntario a lo que podía adherirse si quería mejorar ligeramente sus condiciones económicas, se realizaba en diversas ocasiones. Parece que algunos modelos universitarios foráneos -como es el caso del alemán-, en los que el cuerpo docente se dedicaba exclusivamente al desempeño de sus labores universitarias, sin descuidar las tareas investigadoras, sirvieron como referencia para la configuración de esta nueva figura docente.

Y es que uno de los aspectos en los que se más se insistió a lo largo del articulado de la normativa en cuestión fue en la obligación de dedicación plena a la universidad para todos aquellos que adquirieran la condición de catedrático o agregado a partir de la promulgación de esta Ley. Aun así, nuevamente, si continuamos leyendo la disposición nos encontramos con las pertinentes excepciones, recogiéndose lo siguiente:

«Con objeto de favorecer la enseñanza, y de no privar a la sociedad del concurso de los profesores universitarios, se podrá autorizar por Orden Ministerial la prestación de servicios profesionales a particulares y entidades por el personal docente de las Universidades, aunque se encuentre en régimen de exclusiva dedicación. En estas órdenes se determinarán las condiciones en que podrá ser ejercida dicha actividad profesional, la cual siempre que sea posible se realizará en el ámbito de los respectivos Centros universitarios, con fiscalización y distribución 
económica, que será ordenada por los Centros respectivos bajo la inspección de las autoridades académicas».

Por otra parte, los nuevos agregados podrían ser designados para los distintos cargos universitarios, a excepción de los de rector, vicerrector, decano, vicedecano y director de departamento, aunque se les permitiría que los asumieran de manera interina siempre que no hubiera ningún catedrático para ocuparlos. Además, podrían optar por concurso entre los de disciplina igual o equiparada a las plazas de su categoría vacantes en cualquier universidad, pero no pedir excedencia voluntaria ni solicitar su pase a supernumerario hasta transcurridos dos años de servicio activo (Rubio Mayoral, 2012/13: 220). Un par de años más tarde (1968) se introdujo también la posibilidad de que éstos formasen parte de los tribunales que deberían juzgar tesis doctorales. Dicha modificación en la normativa se llevó a cabo gracias a la mediación de la Universidad de Granada.

\section{Las primeras dotaciones de agregados}

La Ley de 1965 proyectó la dotación de mil nuevas plazas de profesores agregados a cubrir en un plazo de ocho años. Debemos tener en cuenta también que, en las disposiciones transitorias de esta Ley, se estableció el procedimiento para el acceso que consistió en que, durante los primeros cuatro años a partir de su promulgación, el 25\% de las plazas de agregados que se convocaran a concurso-oposición dentro de cada disciplina se haría en turno restringido entre quienes fueran o hubieran sido profesores adjuntos con anterioridad a la promulgación.

A partir del curso 1966/67 comenzaron a ingresar profesores agregados en las universidades españolas o, mejor dicho, en las universidades de Madrid y Barcelona. Porque en un principio allí fueron a parar todas estas plazas, justificándose en el elevado número de alumnos que registraban en sus matrículas, bastante superior a las tasas de otras universidades. Debemos tener en cuenta también que ambas universidades contaban con una oferta de secciones completa en todas sus facultades, aspecto que en otras universidades todavía no se había logrado. Esta condición generaba tanto la atracción de un mayor número de estudiantes como la necesidad de contar con una plantilla docente más amplia.

Pues bien, dos órdenes aprobadas el 30 de mayo de $1966^{8}$ dotaron las primeras plazas de agregados en Madrid y Barcelona, dentro de sus facultades de Filosofía y Letras y Ciencias. En total se convocaron 82 dotaciones de agregados, 33 con destino Barcelona y 49 a Madrid, que se distribuyeron por secciones del modo en que sigue:

\footnotetext{
${ }^{8}$ Orden de 30 de mayo de 1966 por la que se dotan en las Facultades de Filosofía y Letras de las Universidades de Barcelona y Madrid las plazas de profesores agregados que se citan y Orden de 30 de mayo de 1966 por la que se dotan en las Facultades de Ciencias de las Universidades de Barcelona y Madrid las plazas de profesores agregados que se citan, $B O E \mathrm{n}^{\circ} 158$ de 4 de julio de 1966.
} 


\begin{tabular}{|c|c|c|}
\hline Secciones & Universidad de Barcelona & Universidad de Madrid \\
\hline Filologia Clásica & $\begin{array}{l}\text { Literatura griega } \\
\text { Lingüistica griega } \\
\text { Literatura latina } \\
\text { Latin tardio y medieval }\end{array}$ & $\begin{array}{c}\text { Filologia griega } \\
\text { Lingüistica griega } \\
\text { Filologia latina ( } 2 \text { dotaciones) } \\
\text { Lingüistica latina ( } 2 \text { dotaciones) }\end{array}$ \\
\hline Filologia Semítica & Lengua y literatura árabes & Lengua árabe \\
\hline Filologia Románica & $\begin{array}{l}\text { Lengua española } \\
\text { Literatura española }\end{array}$ & $\begin{array}{c}\text { Literatura española } \\
\text { Literatura españolay sus relaciones con la literatura } \\
\text { universal } \\
\text { Lengua española ( } 2 \text { dotaciones) }\end{array}$ \\
\hline Filosofia & $\begin{array}{c}\text { Fundamentos de Filosofia } \\
\text { Historia de los sistemas filosóficos }\end{array}$ & $\begin{array}{l}\text { Fundamentos de Filosofia ( } 2 \text { dotaciones) } \\
\text { Historia de los sistemas filosóficos ( } 2 \text { dotaciones) }\end{array}$ \\
\hline Historia & $\begin{array}{l}\text { Historia antigua, universal y de España } \\
\text { Historia media, universal y de España } \\
\text { Historia moderna, universal y de España } \\
\text { Historia contemporánea, universal y de Espana } \\
\text { Historia del Arte antiguo y medieval } \\
\text { Historia del Arte moderno y contemporáneo } \\
\text { Geografia de España }\end{array}$ & $\begin{array}{c}\text { Historia antigua, universal y de España } \\
\text { Historia media, universal y de España } \\
\text { Historia moderna, universal y de España } \\
\text { Historia contemporánea, universal y de España } \\
\text { Historia del Arte (2 dotaciones) } \\
\text { Historia del Arte medieval, árabe y cristiano } \\
\text { Historia del Arte moderno y contemporáneo } \\
\text { Geografia general y de España (2 dotaciones) }\end{array}$ \\
\hline Total dotaciones & 16 & 25 \\
\hline
\end{tabular}

Tabla I: Primeras dotaciones de profesores agregados en las facultades de Filosofía y Letras de las universidades de Barcelona y Madrid. Elaboración propia a partir de: Orden de 30 de mayo de 1966, $B O E \mathrm{n}^{\circ} 158$ de 4 de julio de 1966.

\begin{tabular}{|c|c|c|}
\hline Secciones & Universidad de Barcelona & Universidad de Madrid \\
\hline Matemáticas & & $\begin{array}{c}\text { Análisis funcional } \\
\text { Análisis de variable real y compleja } \\
\text { Ecuaciones diferenciales } \\
\text { Geometria analitica y diferencial } \\
\text { Topologia }\end{array}$ \\
Investigación operativa
\end{tabular}

Tabla II: Primeras dotaciones de profesores agregados en las facultades de Ciencias de las universidades de Barcelona y Madrid. Elaboración propia a partir de: Orden de 30 de mayo de 1966, $B O E \mathrm{n}^{\circ} 158$ de 4 de julio de 1966. 
Una nueva Orden de 29 de noviembre de $1966^{9}$ completó las plazas citadas en la primera tabla para la Universidad de Barcelona con cuatro nuevas dotaciones (Filología latina, Literatura española, Fundamentos de Filosofía e Historia general del Arte). Unos meses más tarde, por Orden de 11 de enero de $1967^{10}$, se aprobaron nuevas dotaciones en la Facultad de Ciencias de la Universidad de Madrid (en la sección de Matemáticas: Ecuaciones funcionales y Álgebra; en la de Físicas: Termología y Mecánica estadística; Óptica y Estructura de la materia; Electrotecnia y Automática; y en la sección de Geológicas: Petrología y Geoquímica de rocas sedentarias).

Durante los meses sucesivos se fueron constituyendo los tribunales y resolviendo los concursos-oposición para la provisión de estas plazas. En el mes de febrero de 1967 apareció la primera orden que contempló la creación de plazas de agregado en universidades que no fueran sólo Madrid o Barcelona, pero el destino continuaba siendo las facultades de Ciencias y Filosofía y Letras. En concreto, se dotaron plazas en las universidades de Murcia, Oviedo, Salamanca, Santiago, Sevilla, Valencia, Valladolid y Zaragoza. Todas ellas recibieron tres plazas en Ciencias y otras tres en Filosofía y Letras, a excepción de Murcia en donde sólo se dotaron dos en Filosofía y Letras y Santiago con dos plazas en Ciencias ${ }^{11}$.

Sin duda, al contrastar los datos, llama la atención el elevado número de dotaciones que fueron a parar a las universidades madrileña y catalana frente al reducido número que se destinó al resto de centros. Esta cuestión, por una parte, se justifica fácilmente atendiendo a las tasas de matrícula y al número de secciones con las que contaban cada una de esas universidades, muy superior en las primeras, pero, por otra parte, también entra en juego la concentración de recursos que se produjo durante aquellos años en esos centros -prioritariamente Madrid por constituir la Universidad Central- y la falta de atención a las necesidades de otras universidades periféricas o «de provincias», aspecto que se denunció constantemente desde las respectivas juntas de facultad y rectorados.

De cualquier forma, poco a poco los agregados fueron llegando a las facultades. En marzo de 1967 una nueva orden destinó por primera vez plazas a las facultades de Farmacia de las universidades de Madrid, Barcelona, Granada y Santiago. El resto de facultades existentes en el panorama universitario español no recibirían a agregados hasta el siguiente curso (1967/68), creándose las primeras en las facultades de Derecho de Madrid por Orden de 30 de octubre de 1967, en Sevilla por Orden de 17 de noviembre de 1967 o en Granada y Valladolid por Orden de 27 de noviembre de 1967.

\footnotetext{
${ }^{9}$ Orden de 29 de noviembre de 1966 por la que se dotan en la Facultad de Filosofía y Letras de la Universidad de Barcelona las plazas de profesores agregados que se citan, $B O E \mathrm{n}^{\circ} 302$ de 19 de diciembre de 1966.

${ }^{10}$ Orden de 11 de enero de 1967 por la que se dotan en la Facultad de Ciencias de la Universidad de Madrid plazas de profesores agregados, $B O E \mathrm{n}^{\circ} 18$ de 21 de enero de 1967.

${ }^{11}$ Vid. Orden de 24 de febrero de 1967 por la que se dotan plazas de agregados en las Facultades de Ciencias y Filosofía y Letras de las Universidades que se indican, $B O E \mathrm{n}^{\circ} 76$ de 30 de marzo de 1967.
} 
En el caso de Medicina, una orden con idéntica fecha a la anterior aprobó plazas de agregados en Madrid, Santiago de Compostela y Sevilla.

El número de profesores agregados fue muy elevado en universidades como las de Madrid y Barcelona y bastante reducido en el resto de universidades. Sirva como ejemplo ilustrativo la situación de la Universidad de Valencia en el curso 1972/73 (Sanz Díaz, 2002: 278), donde la plantilla docente estaba compuesta por 65 catedráticos (8\%), 14 agregados (2\%), 61 adjuntos (7\%) y 666 profesores no numerarios (83\%). Como vemos, el porcentaje más bajo estaba representado por este tipo de docentes.

Tras realizar este breve repaso por las primeras dotaciones de agregados que llegaron a las universidades españolas, llega el momento de adentrarnos en el último punto de este trabajo a través del cual se realizará una inicial y breve aproximación a las motivaciones que condujeron a la creación del agregado ${ }^{12}$.

\section{Posibles motivaciones en la creación de la figura del agregado}

A partir de la segunda mitad de la década de los sesenta, a raíz del progresivo aumento del alumnado, se emprendió un proceso de proliferación de categorías de docentes subalternos o inferiores a la figura por excelencia o el único docente sensu stricto, el catedrático. En 1965 se dio el primer paso y apareció el estamento del profesor agregado, como un camino o un puesto intermedio entre adjunto y catedrático. En palabras de Carabaña (2003), tomando las reflexiones realizadas por Carlos París, la creación de esta figura podría encuadrarse dentro del proceso de reforma del procedimiento de acceso a cátedra, tratando de que la titularidad represente la culminación de carrera docente con su acumulación de experiencia y posibilidad de una valoración efectiva sobre el ejercicio real de la enseñanza, en lugar de la investidura súbita conseguida en el proceso de la oposición.

De este modo, una de las primeras motivaciones que habría conducido hacia la creación del agregado podría haber sido la aspiración de alcanzar cierto enriquecimiento y reorganización de la estructura del profesorado, aumentando el número de funcionarios. Tal y como menciona el mismo Carabaña (2003), se trataba de construir «piramidalmente» -expresión muy en boga en esta época- la docencia y la investigación. De que el trabajo en equipo reemplazase al individualismo y al aislamiento.

Sale en este punto una cuestión de sumo interés: la ligazón entre el proceso de aparición del agregado y la propuesta de organización departamental de la universidad española. Ambos fenómenos se produjeron al mismo tiempo, en un claro intento por modificar la estructuración de la pirámide académica y la organización de ese profesorado dentro de un ente, el departamento, que otorgaría la posibilidad de constituir líneas de investigación más fuertes y productivas, de desarrollar una

\footnotetext{
${ }^{12}$ Este asunto será objeto particular de futuros estudios. Sirvan las siguientes líneas simplemente para apuntar sucintamente algunas de esas posibles motivaciones.
} 
docencia más coordinada, de que los profesores noveles se sintieran más arropados en su periodo de consolidación definitiva en la carrera docente, de mejorar las relaciones docentes, de propiciar la investigación en equipo frente al individualismo de la cátedra, etc. (Zabalza, 2000: 48).

Pero además de esas posibles motivaciones, personalmente consideramos que este nuevo docente surgió casi como la inevitable acción que Lora Tamayo se vio obligado a poner en marcha ante los ojos de la UNESCO. Recordemos que estamos ante una universidad en la que, con demasiada frecuencia, el candidato a catedrático accedía a ese puesto a través de un tribunal que sabía de antemano «a quién debía seleccionar». Por tanto, a partir de este momento, para ser catedrático había que pasar por la condición previa de agregado, un profesor que se enfrentaba a una oposición que, en teoría, se vendía como «democrática», aunque en realidad el control ideológico y de las familias seguía estando asegurado a través del sistema de pruebas aprobado.

Probablemente, en el Manifiesto por una universidad democrática (1966) encontramos una de las mejores argumentaciones a esa evidente corrupción y a ese cada vez más acusado atraso del modelo universitario español:

«El burocratismo centralista de la política universitaria en general, y, en particular, del sistema de provisión de cátedras, el cual, mientras impide la formación de escuelas científicas y culturales, no cumple con la función de evitar la tendenciosidad. Por el contrario, las oposiciones a cátedras se han convertido durante este periodo en un instrumento de censura intelectual ejercida por la administración misma o a través de la estrategia del dominio de los tribunales de oposición por grupos dominantes políticamente en el Estado».

Por otra parte, otra de las cuestiones que se entrelazan con este asunto es la discusión en torno al tema de la libertad de selección del profesorado por parte de las universidades. El propio Lora Tamayo, en el acto inaugural del curso 1964/65 en la Universidad de Oviedo, aludía a aquélla como una realidad en algunas universidades europeas y americanas, pero aspiración irrealizada en otras muchas. Consideraba aquél que la universidad española todavía no estaba en condiciones de asumir este tipo de sistema, motivo por el cual se había proyectado una solución intermedia a través del sistema de agregados, seleccionados por oposición y entre los que, llegado el momento, se proveerían las cátedras y, por otro lado, libertad de contrata de profesores extraordinarios nacionales o extranjeros que, por su valía notoria, interesase su adscripción temporal a las tareas docentes (El Adelanto, 4 de octubre de 1964).

En definitiva, probablemente la decisión ministerial respondió más a un intento de reconciliación y de «lavado de cara» frente a organismos internacionales, que proclamaban en esos momentos la mejora de las condiciones del profesorado, la dignificación de su labor y el establecimiento de ratios profesor-alumno adecuadas, entre otras cosas, y frente a una sociedad que se mantenía bastante alejada de los problemas universitarios y que comenzaba a reconocer la evidente corrupción del sistema, que a otro tipo de motivaciones. 


\section{Conclusiones}

La aparición del agregado en la universidad resultó finalmente una medida de muy corto alcance y limitado recorrido. Las altas esferas ministeriales creyeron solucionar con ello algunos de los problemas más importantes de la universidad española, pero quizá no fueron conscientes, o sí lo fueron pero no quisieron adoptar otro tipo de medidas, que una reforma tan superficial del sistema, pasando por alto planteamientos enfocados a la reforma del propio modelo universitario vigente, no vendría a cambiar de un plumazo la realidad universitaria del momento.

El Ministerio optó por hacer frente a la expansión del alumnado, muy acusado en la mayor parte de universidades, aunque con especial incidencia en Madrid y Barcelona, mediante una política de contratación masiva de profesorado, no funcionario sino profesorado no numerario (PNN), procedimiento que años más tarde provocaría un grave problema de inestabilidad en el empleo de este personal. En consecuencia, el número de plazas de agregados dotadas no fue muy elevado, en comparación proporcional con el resto de figuras docentes de la universidad, colectivo nuevamente responsable de asumir de forma efectiva el movimiento expansivo de la enseñanza superior vivido durante estos años y especialmente en los setenta.

Además, y a pesar de la dilatada y controvertida concreción de la figura del agregado que se vivió en los debates del Consejo de Rectores, el Ministerio acabó desoyendo la opinión del grueso del colectivo docente, no siempre manifestada del mismo modo en Consejo de Rectores -más conservadora- que en Juntas de Facultad, que abogaba por la mejora de las condiciones del adjunto, al que si se le dotaba de un trabajo estable y permanente, sin someterlo a la dureza de la inestabilidad, y se le retribuía económicamente de manera adecuada, podría desarrollar las labores que ahora se encomendaban al nuevo agregado.

Una importante cantidad de catedráticos nombrados ya en las décadas de los ochenta y noventa pasaron previamente por la condición de agregado. De hecho, la agregaduría fue la tónica general para el acceso a la mayor parte de cátedras a partir de mediados de los sesenta. Lo habitual durante estos años, cuando se trataba de nuevas dotaciones, era precisamente que fueran agregaciones y no cátedras y, en todo caso, muy pronto se empezó a rumorear sobre la conversión de las plazas vacantes de agregados en cátedras y la integración de los agregados en el cuerpo de catedráticos numerarios. Y es que, en realidad, ante la semejanza en sus obligaciones y condiciones, la mayor parte de agregados -algunos tuvieron que esperar bastantes años- sólo ocupaban la plaza pacientemente en espera de dar el salto a la ansiada categoría culmen, la de catedrático.

La desaparición de esta figura del escalafón docente en los ochenta demostró la falta de eficacia de su existencia. La LRU de 1983 intentó simplificar el «caos de la selvática e irracional estructura jerárquica del profesorado, totalmente disfuncional, mediante el establecimiento de cuatro únicas categorías del profesorado y la creación 
de una carrera docente» ${ }^{13}$. Asimismo, buscó la desburocratización del régimen jurídico de dicho profesorado a través de la creación de un estatuto propio del funcionario docente e introduciendo las figuras de asociado y visitante. Por otra parte, estimuló a los agregados a concursar a cátedras de universidades españolas y transformó en plazas de catedráticos todas aquellas agregadurías que en el momento de publicarse dicha Ley estuvieran vacantes o no se encontraran en proceso de concurso-oposición.

En consecuencia, esta normativa aparecida ya en periodo de democracia determinaría la desaparición definitiva de una categoría docente de existencia efímera, surgida como un mero "parcheado de cara a la galería», e ineficaz ante la grave problemática universitaria y ante una situación del cuerpo de profesores que se hacía, cada vez con más claridad, absolutamente insostenible.

\section{Referencias bibliográficas}

BLASCO GIL, Y.; MANCEBO, M. F. (2010). Oposiciones y concursos a cátedra de historia en la Universidad de Franco (1939-1950). València: Universitat de València.

CARABAÑA, J. (2003). La carrera del profesor universitario: cada vez más larga, más Pobre y más precaria. Recuperado de firgoa.usc.es/drupal/files/009.doc, 26 de marzo de 2014.

CARRERAS, J. (2003). Evaluación de la calidad docente y promoción del profesorado (III). Legislación universitaria española: de la Ley de Ordenación de la Universidad Española (1943) a la Ley de Reforma Universitaria (1983). Educación Médica, 6/1, 9-31.

CARRERAS ARES, J. J.; RUIZ CARNICER, M. A. (1991). La Universidad Española bajo el régimen de Franco (1939-1975): Actas del Congreso (Zaragoza, del 8 al 11 de noviembre de 1989). Zaragoza: Instituto Fernando el Católico.

CLARET MIRANDA, J. (2006). El atroz desmoche. La destrucción de la Universidad española por el franquismo, 1936-1945. Barcelona: Crítica.

GONZÁLEZ GÓMEZ, S. (2013). La Universidad de Salamanca durante el franquismo (1956/1968). (Tesis doctoral inédita). Departamento de Teoría e Historia de la Educación. Universidad de Salamanca.

HERNÁNDEZ SANDOICA, E. (2002). La dictadura franquista y la Universidad, 1951-1975. (Con especial atención al caso de Madrid). Cuadernos del Instituto Antonio de Nebrija, 5, 127-156.

HERNÁNDEZ SANDOICA, E.; RUIZ CARNICER, M. A.; BALDÓ LACOMBA, M. (2007). Estudiantes contra Franco (1939-1975): oposición política y movilización juvenil. Madrid: La Esfera de los Libros.

\footnotetext{
${ }^{13}$ Ley orgánica 11/1983, de 25 de agosto, de reforma universitaria, BOE $\mathrm{n}^{\circ} 209$ de 1 de septiembre de 1983.
} 
MARTÍNEZ NEIRA, M. (2003). Los catedráticos de la posguerra. Las oposiciones a cátedra de historia del derecho español en el primer franquismo. Cuadernos del Instituto Antonio de Nebrija de Estudios sobre la Universidad, 6, 135-220.

PELÁEZ, M. J. (2008). Las oposiciones a cátedras de Derecho Romano de 1943 (Álvaro D’ors Pérez-Peix, Faustino Gutiérrez Alviz y Francisco Hernández Tejero ( $1^{\text {a }}$ parte). Revista de estudios histórico-jurídicos [online], 30, 505-537.

RODRÍGUEZ LÓPEZ, C. (2002). La Universidad de Madrid en el primer franquismo. Ruptura y continuidad (1939-1951). Madrid: Biblioteca del Instituto Antonio de Nebrija de estudios sobre la universidad - Editorial Dyckinson.

RUBIO MAYORAL, J. L. (2013). Modelos docentes para el nuevo régimen: estudio normativo desde la política de la Universidad española. Cuestiones Pedagógicas: revista de ciencias de la educación, 22, 203-230.

SANZ DÍAZ, B. (2002). La universidad contra la dictadura. Activismo político y represión, Sanz Díaz, Benito (2002). Rojos y demócratas. Valencia: Artes Gráficas Soler.

SEDHE. (1998). La Universidad en el siglo XX (España e Iberoamérica). X Coloquio de Historia de la Educación. Murcia: Universidad de Murcia.

SINDICATO DEMOCRÁTICO DE LOS ESTUDIANTES DE LA UNIVERSIDAD DE BARCELONA (1966). Manifiesto por una universidad democrática (leído en la Asamblea fundacional del Sindicato democrático de estudiantes de la Universidad de Barcelona el día 9 de marzo de 1966). Realidad, 10.

ZABALZA BERAZA, M. (2000). El papel de los departamentos universitarios en la mejora de la calidad de la docencia. Revista interuniversitaria de formación del profesorado, 38, 47-66.

\section{Correspondencia con la autora}

Sara GONZÁLEZ GÓMEZ

Universidad de las Islas Baleares

Departamento de Pedagogía y Didácticas Específicas

Carretera de Valldemossa, km. 7,5. CP. 07122

Islas Baleares, España

Tel. (34) 971259789

e-mail: sara.gonzalez@uib.es 